OPEN ACCESS

Edited by: Huifang Shang,

Sichuan University, China

Reviewed by: Anastasia Bougea,

National and Kapodistrian University of Athens, Greece Joy Mitra,

Houston Methodist Research Institute, United States

Christina Doriana Orru,

Rocky Mountain Laboratories,

National Institute of Allergy

and Infectious Diseases (NIH),

United States

Feng Luo,

National Institute of Neurological Disorders and Stroke (NIH)

United States

${ }^{*}$ Correspondence:

Nan-nan Yang

xyynn1990@163.com

Hong lu

zzu_luhong@163.com

${ }^{\dagger}$ These authors have contributed equally to this work

Specialty section

This article was submitted to Neurodegeneration, a section of the journal

Frontiers in Neuroscience

Received: 10 December 2021

Accepted: 14 February 2022

Published: 03 March 2022

Citation:

Yang N-n, Sang S-s, Peng $T$ and lu H (2022) SNCA rs3910105 is Associated With Development of Rapid Eye Movement Sleep Behavior

Disorder in Parkinson's Disease.

Front. Neurosci. 16:832550.

doi: 10.3389/fnins.2022.832550

\section{SNCA rs3910105 Is Associated With Development of Rapid Eye Movement Sleep Behavior Disorder in Parkinson's Disease}

\author{
Nan-nan Yang ${ }^{1 * \dagger}$, Shu-shan Sang ${ }^{2}$, Tao Peng ${ }^{1}$ and Hong $\mathrm{lu}^{1 *+}$ \\ 1 Department of Neurology, The First Affiliated Hospital of Zhengzhou University, Zhengzhou, China, ${ }^{2}$ Department \\ of Otolaryngology, The First Affiliated Hospital of Zhengzhou University, Zhengzhou, China
}

Background and Purpose: Rapid eye movement (REM) Rapid eye movement sleep behavior disorder (RBD) is a common non-motor symptom of PD. However, the association between the SNCA rs3910105 genotype and RBD in Parkinson's disease (PD) remains unclear.

Methods: This study used Parkinson's Progression Markers Initiative (PPMI) data and included 270 patients with newly diagnosed PD without RBD who were divided into SNCA rs3910105 C carriers (CC+CT; $n=187)$ and $\Pi$ carriers $(n=83)$. They were followed up for 5 years to identify the development of RBD. To investigate the influence of cerebrospinal fluid (CSF) alpha-synuclein ( $\alpha$-syn) and $\beta$-amyloid 1-42 (A $\left.\beta_{42}\right)$ in the association between rs3910105 and RBD, the patients were additionally classified into "high-level" and "low-level" groups using cutoff values for CSF $\alpha$-syn and A $\beta_{42}$ levels.

Results: At baseline, the rs3910105 C allele group had lower CSF $\alpha$-syn and A $\beta_{42}$ levels than the TT group. During the 5.0-year follow-up, the rs3910105 C allele group had a higher incidence of RBD than the TT group. In the subgroup analyses, the effect of the rs3910105 C allele was not found in the "low-level" group. However, in the "high-level" group, the rs3910105 C allele independently increased the risk of RBD.

Conclusion: The SNCA rs3910105 C allele might be a novel genetic risk factor for RBD development in PD, $\alpha$-syn pathways might have a role in this association and more basic research would be needed to elucidate the mechanism in the future.

Keywords: PPMI, Parkinson's disease, REM sleep behavior disorder, SNCA rs3910105, alpha-synuclein

\section{INTRODUCTION}

Parkinson's disease (PD) is an age-related neurodegenerative disease with a wide range of motor and non-motor symptoms (Chaudhuri et al., 2006; Bloem et al., 2021). Rapid eye movement (REM) Rapid eye movement sleep behavior disorder (RBD) is a common non-motor symptom of PD that is characterized by the loss of normal muscle atonia during REM sleep and involves dreamenacting behavior (Dauvilliers et al., 2018; Miglis et al., 2021). PD patients have a prevalence of RBD symptomatology of approximately 40\% (Romenets et al., 2012; Zhang et al., 2017; Diaconu et al., 2021). It has been suggested that older age and longer disease duration are risk factors for 
RBD in patients with PD (Lee et al., 2010; Romenets et al., 2012). Previous studies found that at baseline, cerebrospinal fluid (CSF) alpha-synuclein ( $\alpha$-syn) and CSF $\beta$-amyloid $1-42$ $\left(\mathrm{A} \beta_{42}\right)$ were significantly lower in PD subjects with RBD (Ba et al., 2018; Pagano et al., 2018). Given that CSF $\alpha$-syn and $\mathrm{A} \beta \beta_{42}$ levels inversely correlate with $\alpha$-syn and $\mathrm{A} \beta$ pathology in the brain (Fagan et al., 2006; Wennström et al., 2013; Gold et al., 2021), this observation may support the notion that cerebral synucleinopathy or amyloidopathy contributes to the development of RBD.

The aggregation of $\alpha$-syn, encoded by the SNCA gene, is central to the pathogenesis of PD (Deng and Yuan, 2014; Meade et al., 2019). The SNCA rs3910105 T/C polymorphism has been shown to alter the risk of developing PD (Goris et al., 2007; Nalls et al., 2014; Campêlo and Silva, 2017). Previous studies showed that rs3910105 was a significant predictor of changes in $A \beta_{42}$, which has been shown to be relevant in PD neurodegeneration (Mollenhauer et al., 2017). Another study revealed that rs3910105 was associated with the annual percentage change in dopamine transporter (DAT) availability, indicating that the rs3910105 single nucleotide polymorphisms (SNPs) might affect the progression of PD (Shin et al., 2019).

However, the relationship between the SNCA rs3910105 C allele and $\mathrm{RBD}$ development in $\mathrm{PD}$ patients remains unclear. Therefore, the aim of this study was to examine the associations between the SNCA rs3910105, CSF $\alpha$-syn and $A \beta_{42}$ levels and the development of RBD in a large group of patients with early PD.

\section{MATERIALS AND METHODS}

\section{Study Design and Patients}

The data were obtained from the Parkinson's Progression Markers Initiative (PPMI) database. The PPMI study is an ongoing international, multicenter, cohort study to identify biomarkers of progression in PD. The study protocol and methodology are available on the PPMI website ${ }^{1}$ and have been published elsewhere (Marek et al., 2018). Patients were recruited between 2010 and 2015 through strict inclusion/exclusion criteria, as previously described.

We downloaded data on PD patients from the PPMI database in December 2020. For this analysis, data up to 5 years of followup were included. We excluded patients who had RBD at the baseline visit or were functionally dependent at or were lost to follow-up in the first year following enrollment. Finally, a total of 270 patients were involved in this analysis.

\section{SNCA rs3910105 Genotyping}

DNA was extracted from the whole blood of the patients according to the study protocol as described in the PPMI biologics manual. All sample were genotyped using the Illumina NeuroX array following the manufacturer's protocol. The Genotyping Analysis Module within Genome Studio was used to analyze data (Nalls et al., 2016).

\footnotetext{
${ }^{1}$ http://ppmi-info.org
}

\section{Ascertainment of Rapid Eye Movement Sleep Behavior Disorder}

The presence of probable RBD was defined based on the RBD Screening Questionnaire (RBDSQ). The RBDSQ comprises 10 items investigating the patient's sleep behavior. The total score is a maximum of 13 points. Only dichotomic responses ("yes" or "no") were allowed. Dream frequency, content, and their relation to nocturnal movements and behavior were assessed by items 14. Item 5 evaluated the presence of self-injuries and injuries to the bed partner while asleep. Item 6 explored nocturnal vocalization, limb movements, complex movements, and objects that fall down around the bed. Items 7 and 8 assessed nocturnal awakenings. Items 9 and 10 investigated the presence of global disturbed sleep and any other neurologic disorder. As previously reported (Berg et al., 2021), an RBDSQ score of 5 was used as a cutoff to differentiate patients with RBD (RBDSQ $\geq 5$ ) from those without RBD (RBDSQ <5). When using RBDSQ, a better sensitivity is reached by using a cut off score of 1 for item 6 (including 6.16.4), sensitivity increases to 0.74 at a reasonable specificity of 0.70 in the PD cohort. (PPMI cohort) (Halsband et al., 2018; Halsband et al., 2018), so RBDSQ $\geq 5$ and item $6 \geq 1$ combined as the cutoff for PD patient with RBD.

\section{Cerebrospinal Fluid Sample Collection and Assessments}

CSF was collected and analyzed for $\alpha$-syn, total tau (t-tau), tau phosphorylated at Thr181 ( $\mathrm{p}$-tau), and $\mathrm{A} \beta_{42}$ as previously described (Pagano et al., 2016, 2018). Briefly, CSF was collected using standardized lumbar puncture procedures. CSF was collected into siliconized polypropylene tubes, and the first 1 to $2 \mathrm{~mL}$ of CSF was sent to the site's local laboratory for routine testing for cell count, total protein level, and glucose level. An additional 15 to $20 \mathrm{~mL}$ of CSF was transferred into15$\mathrm{mL}$ conical polypropylene tubes at room temperature, mixed gently, centrifuged at $2000 \mathrm{~g}$ for $10 \mathrm{~min}$ at room temperature, and transferred into $1.5-\mathrm{mL}$ precooled siliconized polypropylene aliquot tubes followed by immediate freezing on dry ice. The frozen aliquots of CSF were shipped overnight to the PPMI Biorepository Core laboratories on dry ice and then thawed, aliquoted into $0.5-\mathrm{mL}$ siliconized polypropylene tubes, refrozen once, and stored at $-80^{\circ} \mathrm{C}$. Measurements of CSF Ab42, tau, and $\mathrm{p}$-tau were made using the xMAP-Luminex platform with INNOBIA AlzBio3 immunoassay kit-based reagents (FujirebioInnogenetics, Ghent, Belgium) at Penn. Commercially available sandwich type immunoassay kits (BioLegend; formerly Covance) were used to analyze CSF a-syn and CSF hemoglobin levels.

Further information can be found in the PPMI biologics manual (see text footnote 1).

\section{Clinical Evaluation}

Motor symptom severity was assessed with the Movement Disorder Society-sponsored revision of the Unified Parkinson's Disease Rating Scale (MDS-UPDRS) Part III and Hoehn and Yahr (H\&Y) scale, and non-motor symptom burden was assessed with MDS-UPDRS Part I. Global cognitive status was assessed 
using the Montreal Cognitive Assessment (MoCA). Domainspecific neuropsychological performance was measured using 7 standardized instruments. Verbal memory was assessed by the Hopkins Verbal Learning Test, with measures of immediate recall, delayed recognition false alarms, and delayed recognition hits. Verbal fluency was assessed by the semantic fluency test. Processing speed was assessed by the Symbol Digit Modalities Test. Visuospatial abilities were assessed by the Benton Judgment of Line Orientation Test. Executive functioning was assessed by the letter-number sequencing test. Depressive symptoms were assessed with the short version of the Geriatric Depression Scale (15-item GDS). Anxiety was assessed with Spielberger's State-Trait Anxiety Inventory (STAI). Autonomic dysfunction was assessed with the Scales for Outcomes in Parkinson's Disease-Autonomic (SCOPA-AUT). Sleep disturbances were assessed with the Epworth Sleepiness Scale. The Questionnaire for Impulsive Compulsive Disorders in $\mathrm{PD}$ was used to assess impulsive-compulsive disorders and other compulsive behaviors. Olfactory dysfunction was measured by the University of Pennsylvania Smell Identification Test (UPSIT). Clinical evaluation was performed in the "off" treatment state.

\section{Statistical Analysis}

Calculations were performed with SPSS 25.0 (SPSS Inc., Chicago, IL, United States) and $\mathrm{R}$ version 3.6.2 (R Foundation for Statistical Computing, Vienna, Austria). Data are presented as the mean \pm standard deviation. Data normality was assessed with the use of the Shapiro-Wilk test. Baseline characteristics were compared with Student's $t$-tests continuous variables and chi-square tests or Fisher's exact tests for categorical variables, as appropriate. We used the Kaplan-Meier method to compare the cumulative incidence of RBD between the groups. Cox proportional hazards regression models were used for an adjusted analysis of the relationship of the SNCA rs3910105 allele with $\mathrm{RBD}$, and hazard ratios (HRs) and 95\% confidence intervals (CIs) were calculated. All baseline variables with $p$-values less than 0.2 in the univariable Cox models were included in subsequent multivariable Cox models with a backward elimination procedure $(p$-value removal $=0.1)$. All $p$-values were two-sided, and a $p$-value of less than 0.05 was regarded as statistically significant. As for the cut off in the subgroup analysis, we used an optimum cutoff value for the CSF $\alpha$-syn and $A \beta_{42}$ levels using the $\mathrm{R}$ package "MaxStat," which selects the cutoff value producing the maximum log-rank score. To increase stability of our findings, we also used the 25 th and 50 th percentile cutoff values of the CSF $A \beta_{42}$ levels. All $p$-values were two-sided, and a $p$-value of less than 0.05 was regarded statistically significant.

\section{RESULTS}

\section{Demographic and Clinical Features}

Among the 270 patients [age $(61.5 \pm 9.7)$; men, $(66 \%)$ ] included in this study, $186(68.8 \%)$ were SNCA rs3910105 C carriers, and $84(31.2 \%)$ were TT carriers. The baseline characteristics of the two groups are depicted in Table 1. There were no significant differences between-group in age, sex, disease duration, age at onset or MDS-UPDRS motor, tremor, postural instability and gait difficulties (PIGD), MoCA, GDS, or STAI scores.

However, the SNCA rs3910105 C allele group had lower CSF $\alpha$-syn levels $(1448 \pm 592.6 \mathrm{pg} / \mathrm{ml}$ vs. $1601 \pm 668.5 \mathrm{pg} / \mathrm{ml}$; $p=0.019)$ (Figure 1A), lower CSF A $\beta_{42}$ levels $(870.4 \pm 370 \mathrm{pg} / \mathrm{ml}$ vs. $965.7 \pm 377.3 \mathrm{pg} / \mathrm{ml} ; p=0.015$ ) (Figure 1B), lower CSF t-tau

TABLE 1 | Baseline characteristics in the rs3910105 C allele group and TT groups.

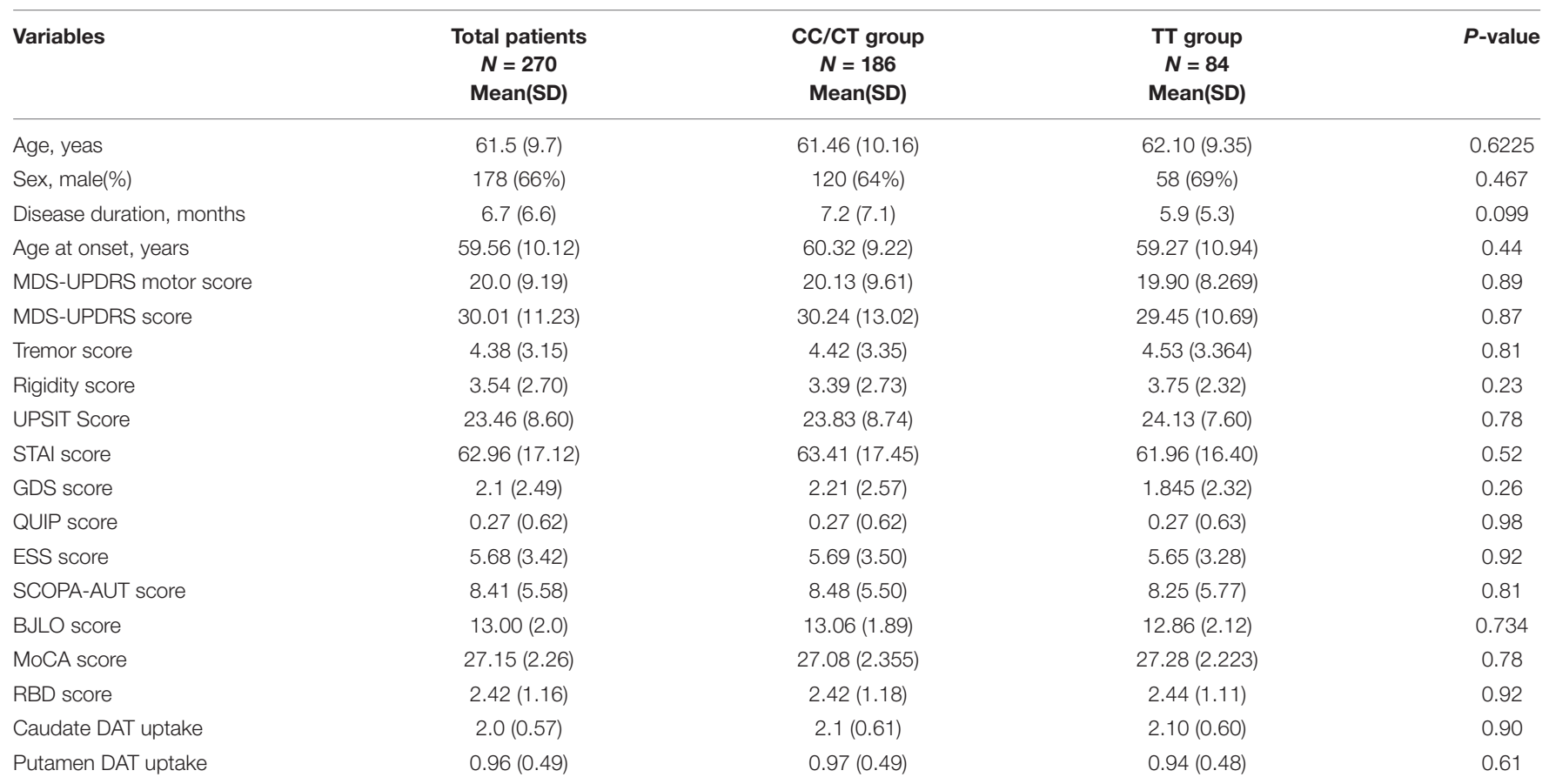



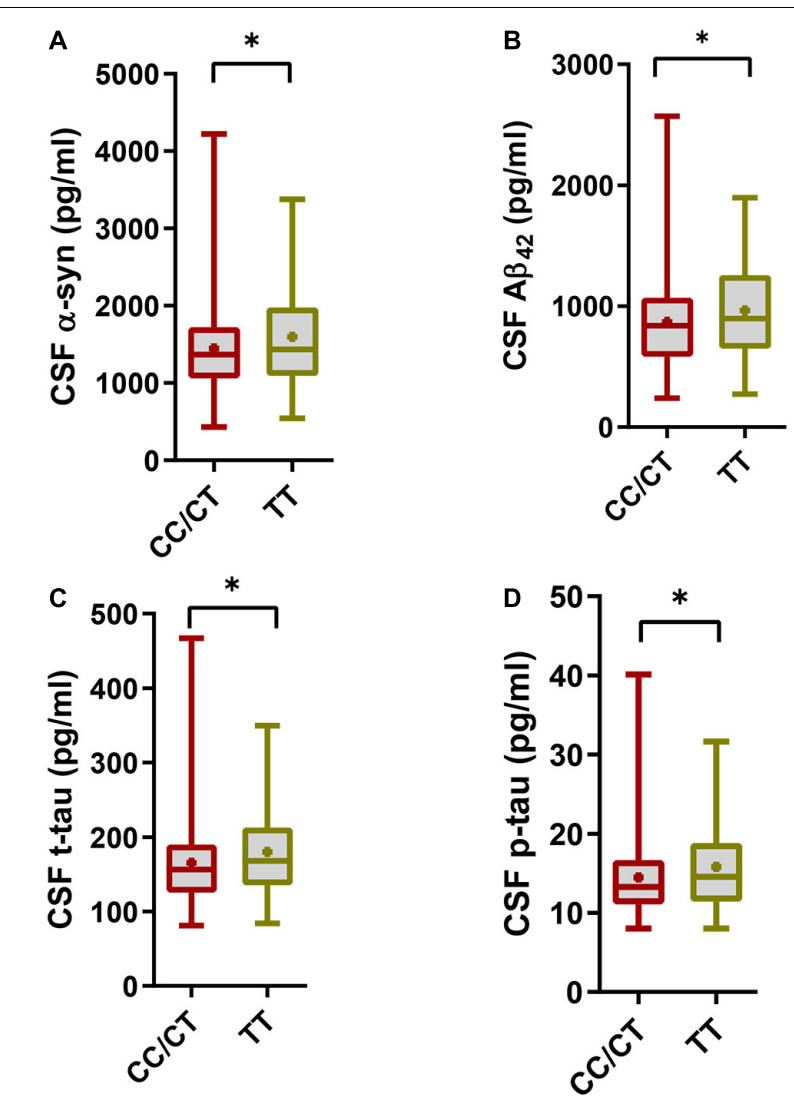

FIGURE 1 | Cerebrospinal fluid (CSF) pathology in the SNCA rs3910105 C allele group and $\Pi$ group. (A) Difference in CSF $\alpha$-syn levels between the rs3910105 C allele and TT groups [Unpaired $t$-test(two-tailed), $t=2.38$, $p=0.019]$; (B) differences in CSF A $\beta_{42}$ levels between the rs3910105 C allele and $T$ groups [Unpaired $t$-test(two-tailed), $t=2.43, p=0.015$ ]; (C) differences in CSF t-tau levels between the rs3910105 $\mathrm{C}$ allele and $\Pi \mathrm{T}$ groups [Unpaired $t$-test(two-tailed), $t=2.47, p=0.012$ ]; (D) differences in CSF p-tau levels between the rs3910105 $\mathrm{C}$ allele and $\mathrm{TT}$ groups [Unpaired $t$-test(two-tailed), $t=2.31, p=0.02]$. ${ }^{*} P<0.05$.

levels $(165.3 \pm 55.43 \mathrm{pg} / \mathrm{ml}$ vs. $180 \pm 59.82 \mathrm{pg} / \mathrm{ml} ; p=0.012)$ (Figure 1C) and lower CSF p-tau levels (14.48 \pm 5.124 vs. $15.80 \pm 5.659, p=0.02$ ) (Figure 1D) than the TT group.

\section{Rs3910105 and the Development of Rapid Eye Movement Sleep Behavior Disorder}

During the follow-up, a total of 129 (47.7\%) patients developed $\mathrm{RBD}$, and the cumulative incidence of $\mathrm{RBD}$ was 18.1, 37.7, and $43.7 \%$ at the 1-, 2- and 4-year follow-ups, respectively (Figure 2A). At baseline, those patients who later developed RBD had a higher prevalence of the SNCA rs3910105 C allele, higher MDS-UPDRS total and motor scores and lower CSF A $\beta_{42}$ levels than those who did not develop RBD (Table 2). There were 100 patients (53.4\%) in the rs3910105 C allele group and 29 patients (34.5\%) in the TT group who developed RBD during the followup period. Kaplan-Meier estimates showed that the rs3910105 C allele group had a significantly higher incidence of RBD than the TT group $(p=0.0068)$ (Figure 2B).
The results of the Cox regression analyses are shown in Table 3. In the univariable Cox regression analyses and multivariable Cox analyses, the rs3910105 C allele and MDSUPDRS scores were significantly related to RBD.

\section{Subgroup Analyses}

Although the baseline levels of CSF $\alpha$-syn and CSF A $\beta_{42}$ could not be related to the future incidence of RBD based on the Cox regression analysis data, as shown in Figure 1, we knew that CSF $\alpha$-syn and CSF $A \beta_{42}$ levels were much lower in the rs3910105 C allele group than in the TT group. Since the RBD development rate was much higher in the $C$ allele group than in the TT group, we speculated that lower baseline CSF $\alpha$-syn and CSF A $\beta_{42}$ might also contribute to the higher incidence of RBD. We divided the patients into two groups based on the CSF $\alpha$-syn and CSF $\mathrm{A} \beta_{42}$ levels.

The cutoff value of CSF $\alpha$-syn in relation to $\mathrm{RBD}$ for producing the maximum log rank score was $805 \mathrm{pg} / \mathrm{ml}$. When the patients were divided by this cutoff value, the "low-level" group (17/23 patients [74\%]) had a significantly higher incidence of RBD than the "high-level" group (106/247 patients [43\%]; $p=0.0083$ ) (Figure 3A).

Furthermore, in the "low-level" group, there was no significant difference in the development of RBD between the rs3910105 C carriers and TT group $(p=0.97)$ (Figure 3B). However, in the "high-level" group, the cumulative incidence of RBD was significantly higher in the rs3910105 C carriers than in the noncarriers $(p=0.028)$ (Figure 3C).

Regarding CSF $A \beta_{42}$ levels, the cutoff value of CSF $A \beta_{42}$ in relation to $\mathrm{RBD}$ for producing the maximum log rank score was $385 \mathrm{pg} / \mathrm{ml}$. When the patients were divided by this cutoff value, the "low-level" group (18/24 patients [75\%]) had a significantly higher incidence of RBD than the "high-level" group (104/246 patients [42\%]; $p=0.043$ ) (Figure 4A).

In the "low-level" group, there was no significant difference in the development of RBD between the rs3910105 C carriers and TT group $(p=0.97)$ (Figure $4 \mathbf{B})$. However, in the "highlevel" group, the cumulative incidence of RBD was significantly higher in the rs3910105 $\mathrm{C}$ carriers than in the non-carriers $(p=0.022)$ (Figure 4C).

\section{DISCUSSION}

This study investigated the relationship of the SNCA rs3910105 $\mathrm{C}$ allele with the development of RBD in patients with early PD using the PPMI cohort. Our results showed that the SNCA rs3910105 C allele was associated with faster development of RBD in PD patients, suggesting that the SNCA rs3910105 C allele was a novel genetic risk factor for RBD. To our knowledge, this was the first study to demonstrate the association of SNCA rs3910105 and RBD development in PD patients.

Studies have shown that rs3910105 was associated with CSF $\mathrm{A} \beta_{42}$ levels (Mollenhauer et al., 2017), which was consistent with our results that CSF pathology (including $\alpha$-syn, $A \beta_{42}$, t-tau and p-tau) in the SNCA rs3910105 C allele group was much lower than that in the TT group. Furthermore, studies have found 


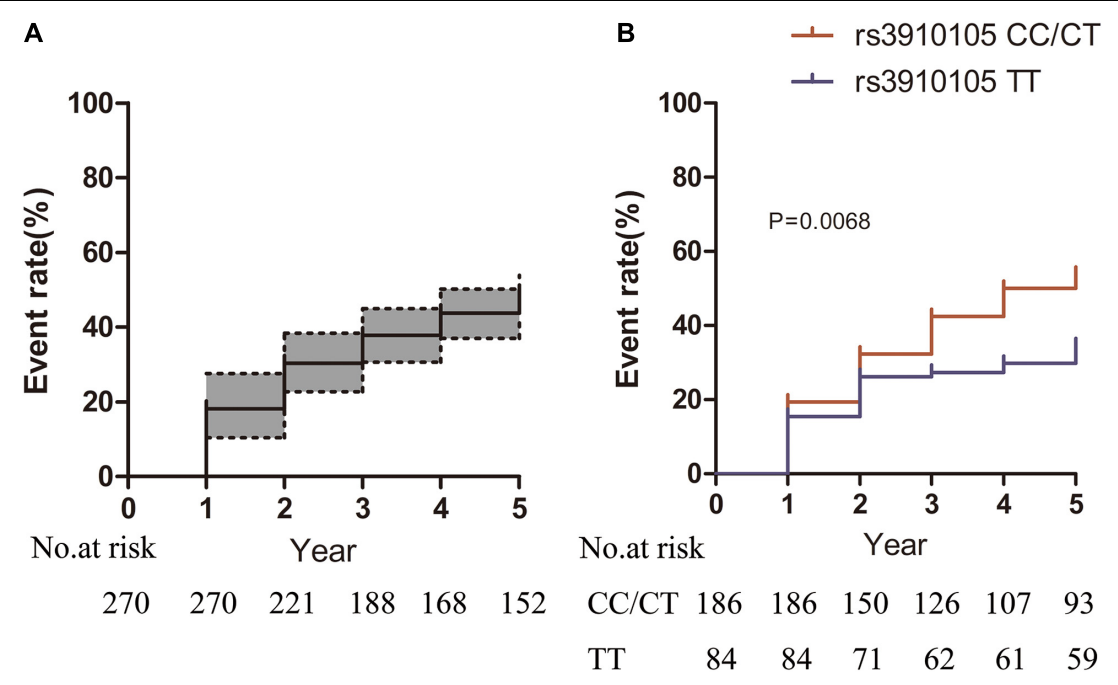

FIGURE 2 | Kaplan-Meier estimates showing the cumulative risk of RBD in (A) the total sample of patients and (B) the rs3910105 C carrier group and $7 T$ group. In panel (A), dashed lines represent the 95\% confidence interval range. In panel (B), the red line indicates the rs3910105 C allele group, and the blue line indicates the Tा group.

TABLE 2 | Baseline characteristics of PD patients who later developed RBD or without RBD during.

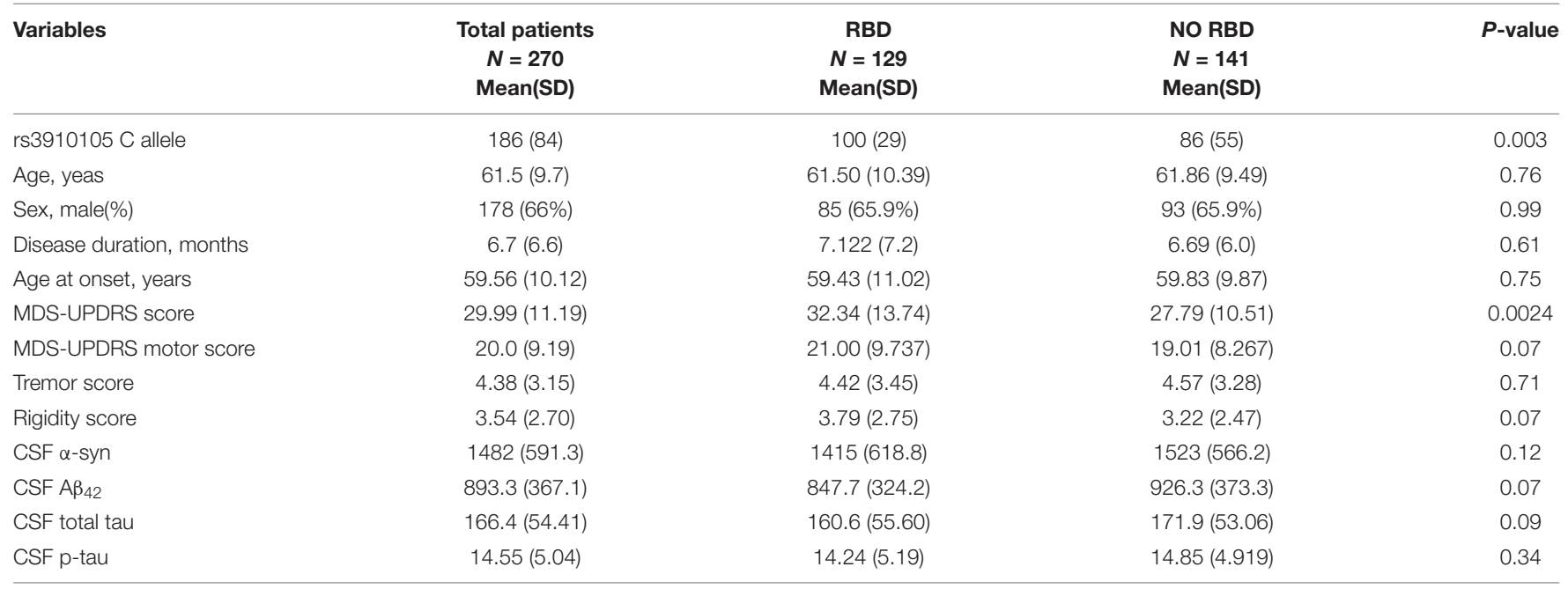

TABLE 3 | Results of the Cox regression analyses for the predictors of RBD.

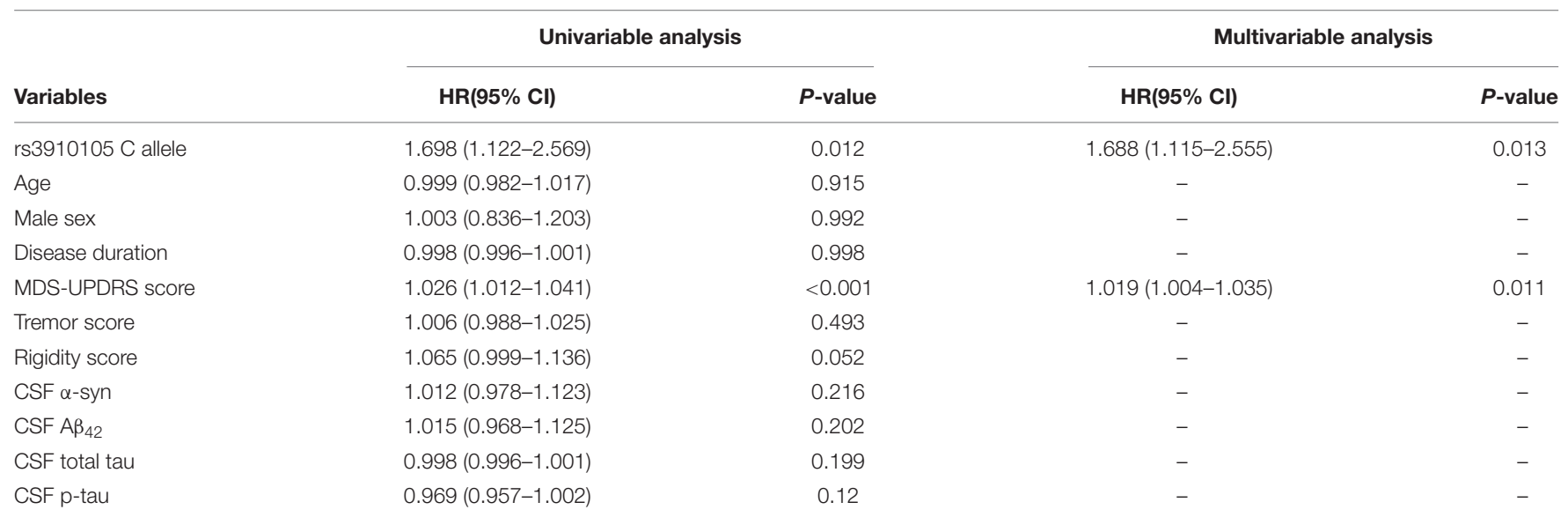




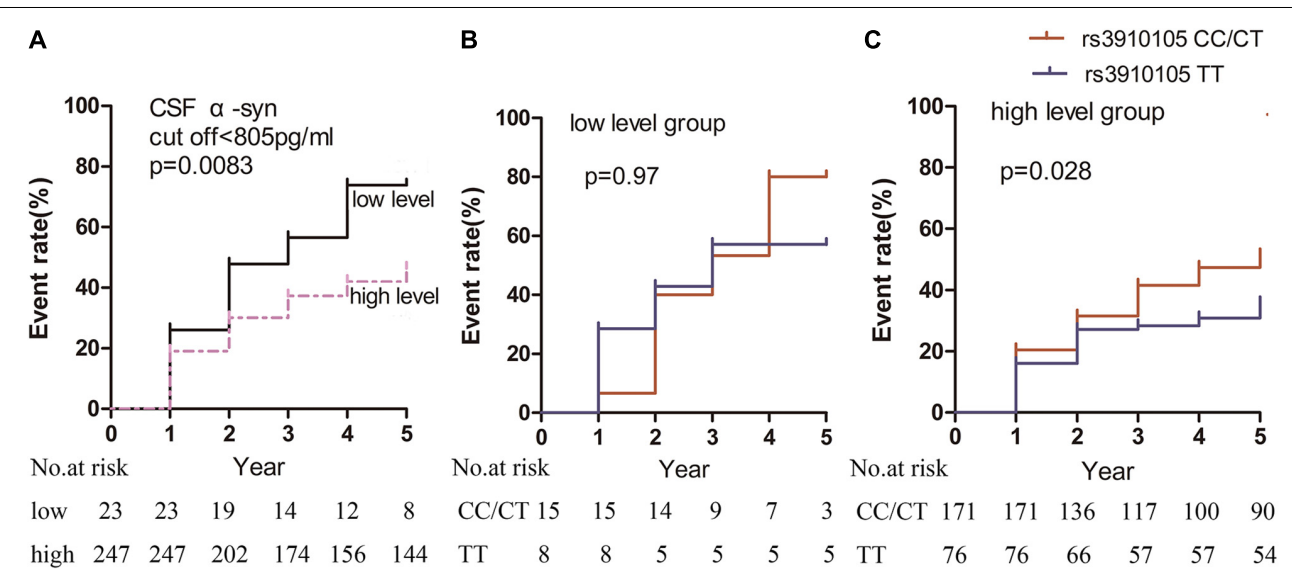

FIGURE 3 | Kaplan-Meier estimates showing the cumulative risk of RBD based on $\alpha$-syn levels in the (A) "low-level" and "high-level" groups, (B) rs3910105 C carriers and non-carriers in the "low-level" group, and (C) rs3910105 C carriers and non-carriers in the "high-level" group. In panel (A), the black line indicates the "low-level" group, and the dashed purple line indicates the "high-level" group. In panels (B,C), red lines indicate rs3910105 C carriers, and blue lines indicate non-carriers.
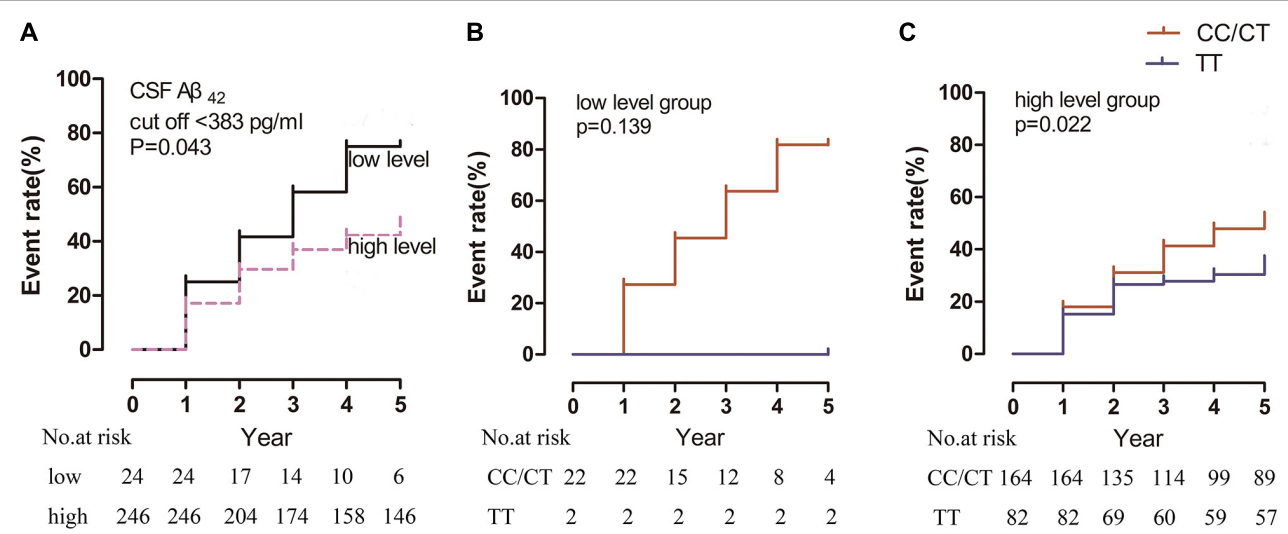

FIGURE 4 | Kaplan-Meier estimates showing the cumulative risk of RBD based on A $\beta_{42}$ levels in the (A) "low-level" and "high-level" groups, (B) rs3910105 C and non-carriers in the "low-level" group, and (C) rs3910105 C carriers and non-carriers in the "high-level" group. In panel (A), the black line indicates the "low-level" group, and the dashed purple line indicates the "high-level" group. In panels (B,C), red lines indicate rs3910105 C carriers, and blue lines indicate non-carriers.

that CSF $\alpha$-syn and $A \beta_{42}$ were significantly lower in PD subjects with RBD (Ba et al., 2018; Pagano et al., 2018). Therefore, we speculated that the SNCA rs3910105 C allele group might have a higher incidence of RBD development due to the $\mathrm{C}$ allele group's lower CSF pathology, which may contribute to the development of RBD. The Cox regression analysis results confirmed our hypothesis. Based on the results, the SNCA rs3910105 C allele and MDS-UPDRS total scores were related to RBD incidence. However, none of the CSF pathologies contributed to RBD development according to our Cox model, which might be related to a limited number of patients. Since there came a trend that the baseline CSF pathology levels ( $\alpha$-syn and $A \beta_{42}$ ) were lower in $\mathrm{PD}$ patients who later developed RBD, we did further subgroup analysis that divided the PD patients into low and high groups based on the baseline CSF pathology levels ( $\alpha$-syn and $A \beta_{42}$ ), and it turned out that the "low-level" group did have a significantly higher incidence of RBD than the "high-level" group. However we found the effect of the rs3910105 C allele was not in the "low-level" group, which was possible considering the limited number of this group and a low CSF pathology baseline level that they already had, the rs3910105 C allele effect to keep the low level of CSF pathology and then increase the risk of RBD development as PD progress might not work. While in the "high-level" group, the effect was still there, the rs3910105 C allele prevalence contributed to a lower CSF $\alpha$-syn and $A \beta_{42}$ both at baseline and during the follow up study, thus increasing the risk of RBD during disease progress. which indirectly showed an association between CSF $\alpha$-syn and $A \beta_{42}$ levels and RBD incidence.

Rs3910105 is an intronic variant in the SNCA gene and is located in the CpG island of SNCA (Tagliafierro and Chiba-Falek, 2016; Funahashi et al., 2017). The SNCA gene encodes $\alpha$-syn, which consists of 140 amino acid residues and is involved in the etiology of PD and other neurodegenerative diseases (Attwood et al., 2002; Mata et al., 2010). Since the CpG island methylation level was able to regulate gene expression (Attwood et al., 2002; Moore et al., 2013), the rs3910105 SNP somehow regulates 


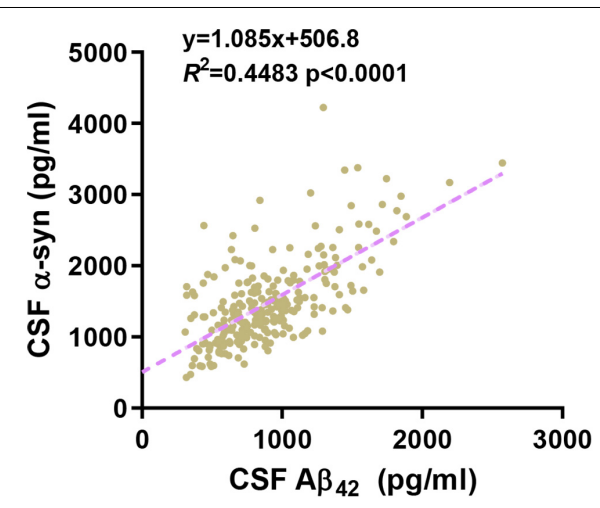

FIGURE 5 | Cerebrospinal fluid (CSF) $\alpha$-syn levels correlated with $A \beta_{42}$ levels.

different SNCA transcript targets. A recent transcript-level linear modeling study based on the PPMI database showed that the C allele of rs3910105 was able to upregulate SNCA gene expression (Koks et al., 2021). Furthermore, another study found that the rs3910105 genotype showed negative correlations with DAT availability in the putamen with SNCA transcripts (Shin et al., 2019), indicating that the rs3910105 C allele was able to impact $\alpha$-syn expression and accumulation and even the progression of PD development.

Previous studies have demonstrated that RBD was a strong predictor for the development of synucleinopathy, most frequently PD or dementia with Lewy bodies (DLB) (Högl et al., 2018). However, the incidence of RBD development in early $\mathrm{PD}$ patients and the underlying pathogenesis have not received much attention. In our longitudinal observational study, we shed light on the role of rs3910105 in RBD development in early PD patients, which might occur through the contribution of cerebral synucleinopathy. Even though the Cox model did not provide a direct correlation between CSF pathology and RBD development in PD patients, we obtained more information through a subgroup analysis in which a lower level of CSF $\alpha$-syn was related to a higher incidence of RBD. As we previously cited, rs3910105 was a predictor of changes in $\mathrm{A} \beta_{42}$, but the underlying mechanism remains unknown. Studies on the impact of rs3910105 on $A \beta_{42}$ are limited, and elucidation of this relationship needs more investigation. Studies have shown that CSF $\alpha$-syn is positively correlated with $\mathrm{A} \beta_{42}$ in PD participants (Buddhala et al., 2015). We also performed correlation analysis in our study and found that CSF $\alpha$-syn correlated with $A \beta_{42}$ $\left(R^{2}=0.44\right)$ (Figure 5), suggesting a pathophysiologic connection between the metabolism of these proteins in PD.

There were some limitations to note in this study. First, the presence of RBD was based on results from the RBDSQ, while a definite diagnosis of RBD requires polysomnography (PSG). Because PSG is expensive and requires a skilled examiner, the RBDSQ is used more frequently in large cohort follow-up studies. In this context, the RBD data in the current study might have been misestimated. Second, even though using the subgroup analysis, we indirectly showed an association between baseline CSF $\alpha$-syn and $A \beta_{42}$ levels and RBD incidence, but the Cox model did not work. We could only speculate this association may related with contributions made by rs3910105 C allele.

In conclusion, our results suggest that the rs3910105 $\mathrm{C}$ allele is associated with an increased risk of RBD development in $\mathrm{PD}$, and $\alpha$-syn pathways might have a role in this association Further studies need to be conducted to validate our findings and to reveal the precise mechanisms underlying the influence of the rs3910105 C allele in the development of RBD in patients with PD.

\section{DATA AVAILABILITY STATEMENT}

The datasets presented in this study can be found in online repositories. The names of the repository/repositories and accession number(s) can be found in the article/supplementary material.

\section{ETHICS STATEMENT}

The studies involving human participants were reviewed and approved by Parkinson's Progression Markers Initiative (PPMI). The patients/participants provided their written informed consent to participate in this study.

\section{AUTHOR CONTRIBUTIONS}

N-NY conceived and designed the project and prepared the writing of the first draft. S-SS designed and executed most of the statistical analysis and reviewed the first draft. TP executed some statistical analysis and prepared the figures and tables. $\mathrm{Hl}$ conceived and designed the project, make the critique, and reviewed the final draft. All authors contributed to the article and approved the submitted version.

\section{FUNDING}

This study was also supported by grants from China Postdoctoral Science Foundation funded project (No. 2021M702945) and Joint Co-construction Project of Henan Medical Science and Technology Research (No. LHGJ20210300) to N-NY, the National Natural Science Foundation of China (No. 82101940 to S-SS and No. 81971175 to $\mathrm{Hl})$.

\section{ACKNOWLEDGMENTS}

Data used in the preparation of this article were obtained from the Parkinson's Progression Markers Initiative (PPMI) database (www.ppmi-info.org/data). For up-to-date information on the study, visit www.ppmi-info.org. PPMI (a publicprivate partnership) was funded by the Michael J. Fox Foundation for Parkinson's Research and multiple funding partners, including AbbVie, Avid, Biogen, Bristol-Myers Squibb, Covance, GE Healthcare, Genentech, GlaxoSmithKline, Lilly, Lundbeck, Merck, Meso Scale Discovery, Pfizer, Piramal, Roche, Servier, Teva, and UCB. 


\section{REFERENCES}

Attwood, J., Yung, R., and Richardson, B. (2002). DNA methylation and the regulation of gene transcription. Cell. Mol. Life Sci. CMLS 59, 241-257. doi: 10.1007/s00018-002-8420-z

Ba, M., Yu, G., Kong, M., Liang, H., and Yu, L. (2018). CSF A $\beta$ level is associated with cognitive decline in early Parkinson's disease with rapid eye movement sleep behavior disorder. Trans. Neurodegenerat. 7:22. doi: 10.1186/s40035-0180129-5

Berg, D., Borghammer, P., Fereshtehnejad, S., Heinzel, S., Horsager, J., Schaeffer, E., et al. (2021). Prodromal parkinson disease subtypes - key to understanding heterogeneity. Nat. Rev. Neurol. 17, 349-361. doi: 10.1038/s41582-021-00486-9

Bloem, B., Okun, M., and Klein, C. (2021). Parkinson's disease. Lancet (London, England) 397, 2284-2303. doi: 10.1016/s0140-6736(21)00 218-x

Buddhala, C., Campbell, M., Perlmutter, J., and Kotzbauer, P. (2015). Correlation between decreased CSF $\alpha$-synuclein and $A \beta_{1-42}$ in parkinson disease. Neurobiol. Aging 36, 476-484. doi: 10.1016/j.neurobiolaging.2014.07.043

Campêlo, C., and Silva, R. (2017). Genetic variants in SNCA and the risk of sporadic Parkinson's disease and clinical outcomes: a review. Parkinson's Dis. 2017:4318416. doi: 10.1155/2017/4318416

Chaudhuri, K., Martinez-Martin, P., Schapira, A., Stocchi, F., Sethi, K., Odin, P., et al. (2006). International multicenter pilot study of the first comprehensive self-completed nonmotor symptoms questionnaire for Parkinson's disease: the NMSQuest study. Movement Dis. Off. J. Movement Dis. Soc. 21, 916-923. doi: $10.1002 / \mathrm{mds} .20844$

Dauvilliers, Y., Schenck, C. H., Postuma, R. B., Iranzo, A., Luppi, P. H., Plazzi, G., et al. (2018). REM sleep behaviour disorder. Nat. Rev. Dis. Primers 4:19. doi: 10.1038/s41572-018-0016-5

Deng, H., and Yuan, L. (2014). Genetic variants and animal models in SNCA and parkinson disease. Ageing Res. Rev. 15, 161-176. doi: 10.1016/j.arr.2014.04.002

Diaconu, Ş, Falup-Pecurariu, O., Ţînţ, D., and Falup-Pecurariu, C. (2021). REM sleep behaviour disorder in Parkinson's disease (review). Exp. Ther. Med. 22:812. doi: 10.3892/etm.2021.10244

Fagan, A. M., Mintun, M. A., Mach, R. H., Lee, S. Y., Dence, C. S., Shah, A. R., et al. (2006). Inverse relation between in vivo amyloid imaging load and cerebrospinal fluid Abeta42 in humans. Ann. Neurol. 59, 512-519. doi: 10.1002/ ana. 20730

Funahashi, Y., Yoshino, Y., Yamazaki, K., Mori, Y., Mori, T., Ozaki, Y., et al. (2017). DNA methylation changes at SNCA intron 1 in patients with dementia with lewy bodies. Psychiatry Clin. Neurosci. 71, 28-35. doi: 10.1111/pcn.1 2462

Gold, B., Shao, X., Sudduth, T., Jicha, G., Wilcock, D., Seago, E., et al. (2021). Water exchange rate across the blood-brain barrier is associated with CSF amyloid$\beta 42$ in healthy older adults. Alzheimer's Dementia J. Alzheimer's Assoc. 17, 2020-2029. doi: 10.1002/alz.12357

Goris, A., Williams-Gray, C., Clark, G., Foltynie, T., Lewis, S., Brown, J., et al. (2007). Tau and alpha-synuclein in susceptibility to, and dementia in, Parkinson's disease. Ann. Neurol. 62, 145-153. doi: 10.1002/ana.21192

Halsband, C., Zapf, A., Sixel-Doring, F., Trenkwalder, C., and Mollenhauer, B. (2018). The REM sleep behavior disorder screening questionnaire is not valid in de novo Parkinson's disease. Mov. Disord Clin. Pract. 5, 171-176. doi: 10. $1002 / \mathrm{mdc} 3.12591$

Högl, B., Stefani, A., and Videnovic, A. (2018). Idiopathic REM sleep behaviour disorder and neurodegeneration - an update. Nat. Rev. Neurol. 14, 40-55. doi: 10.1038/nrneurol.2017.157

Koks, S., Pfaff, A., Bubb, V., and Quinn, J. (2021). Transcript variants of genes involved in neurodegeneration are differentially regulated by the APOE and MAPT haplotypes. Genes 12:423. doi: 10.3390/genes 12030423

Lee, J., Kim, K., Shin, H., and Sohn, Y. (2010). Factors related to clinically probable REM sleep behavior disorder in parkinson disease. Parkinsonism Related Dis. 16, 105-108. doi: 10.1016/j.parkreldis.2009.08.005

Marek, K., Chowdhury, S., Siderowf, A., Lasch, S., Coffey, C., Caspell-Garcia, C., et al. (2018). The Parkinson's progression markers initiative (PPMI) establishing a PD biomarker cohort. Ann. Clin. Trans. Neurol. 5, 1460-1477. doi: $10.1002 / \operatorname{acn} 3.644$
Mata, I. F., Shi, M., Agarwal, P., Chung, K. A., Edwards, K. L., Factor, S. A., et al. (2010). SNCA variant associated with parkinson disease and plasma alphasynuclein level. Arch. Neurol. 67, 1350-1356. doi: 10.1001/archneurol.2010. 279

Meade, R., Fairlie, D., and Mason, J. (2019). Alpha-synuclein structure and Parkinson's disease - lessons and emerging principles. Mol. Neurodege. 14:29. doi: 10.1186/s13024-019-0329-1

Miglis, M., Adler, C., Antelmi, E., Arnaldi, D., Baldelli, L., Boeve, B., et al. (2021). Biomarkers of conversion to $\alpha$-synucleinopathy in isolated rapid-eyemovement sleep behaviour disorder. Lancet Neurol. 20, 671-684. doi: 10.1016/ s1474-4422(21)00176-9

Mollenhauer, B., Caspell-Garcia, C., Coffey, C., Taylor, P., Shaw, L., Trojanowski, J., et al. (2017). Longitudinal CSF biomarkers in patients with early parkinson disease and healthy controls. Neurology 89, 1959-1969. doi: 10.1212/wnl. 0000000000004609

Moore, L., Le, T., and Fan, G. (2013). DNA methylation and its basic function. Neuropsychopharmacol. Off. Publi. Am. College Neuropsychopharmacol. 38, 23-38. doi: 10.1038/npp.2012.112

Nalls, M., Keller, M., Hernandez, D., Chen, L., Stone, D., and Singleton, A. (2016). Baseline genetic associations in the Parkinson's progression markers initiative (PPMI). Mov. Dis. Off. J. Mov. Dis. Soc. 31, 79-85. doi: 10.1002/mds.26374

Nalls, M., Pankratz, N., Lill, C., Do, C., Hernandez, D., Saad, M., et al. (2014). Largescale meta-analysis of genome-wide association data identifies six new risk loci for Parkinson's disease. Nat. Genet. 46, 989-993. doi: 10.1038/ng.3043

Pagano, G., De Micco, R., Yousaf, T., Wilson, H., Chandra, A., and Politis, M. (2018). REM behavior disorder predicts motor progression and cognitive decline in parkinson disease. Neurology 91, e894-e905. doi: 10.1212/wnl. 0000000000006134

Pagano, G., Ferrara, N., Brooks, D., and Pavese, N. (2016). Age at onset and parkinson disease phenotype. Neurology 86, 1400-1407. doi: 10.1212/wnl. 0000000000002461

Romenets, S., Gagnon, J., Latreille, V., Panniset, M., Chouinard, S., Montplaisir, J., et al. (2012). Rapid eye movement sleep behavior disorder and subtypes of Parkinson's disease. Mov. Dis. Off. J. Mov. Dis. Soc. 27, 996-1003. doi: 10.1002/ mds. 25086

Shin, S., Kim, K., Lee, J., Kim, E., Kim, S., Kim, I., et al. (2019). Effect of singlenucleotide polymorphisms on decline of dopamine transporter availability in Parkinson's disease. J. Clin. Neurol. (Seoul, Korea) 15, 102-107. doi: 10.3988/ jcn.2019.15.1.102

Tagliafierro, L., and Chiba-Falek, O. (2016). Up-regulation of SNCA gene expression: implications to synucleinopathies. Neurogenetics $17,145-157$. doi: 10.1007/s10048-016-0478-0

Wennström, M., Surova, Y., Hall, S., Nilsson, C., Minthon, L., Boström, F., et al. (2013). Low CSF levels of both $\alpha$-synuclein and the $\alpha$-synuclein cleaving enzyme neurosin in patients with synucleinopathy. PLoS One 8:e53250. doi: 10.1371/ journal.pone.0053250

Zhang, J., Xu, C., and Liu, J. (2017). Meta-analysis on the prevalence of REM sleep behavior disorder symptoms in Parkinson's disease. BMC Neurol. 17:23. doi: 10.1186/s12883-017-0795-4

Conflict of Interest: The authors declare that the research was conducted in the absence of any commercial or financial relationships that could be construed as a potential conflict of interest.

Publisher's Note: All claims expressed in this article are solely those of the authors and do not necessarily represent those of their affiliated organizations, or those of the publisher, the editors and the reviewers. Any product that may be evaluated in this article, or claim that may be made by its manufacturer, is not guaranteed or endorsed by the publisher.

Copyright (c) 2022 Yang, Sang, Peng and lu. This is an open-access article distributed under the terms of the Creative Commons Attribution License (CC BY). The use, distribution or reproduction in other forums is permitted, provided the original author(s) and the copyright owner(s) are credited and that the original publication in this journal is cited, in accordance with accepted academic practice. No use, distribution or reproduction is permitted which does not comply with these terms. 